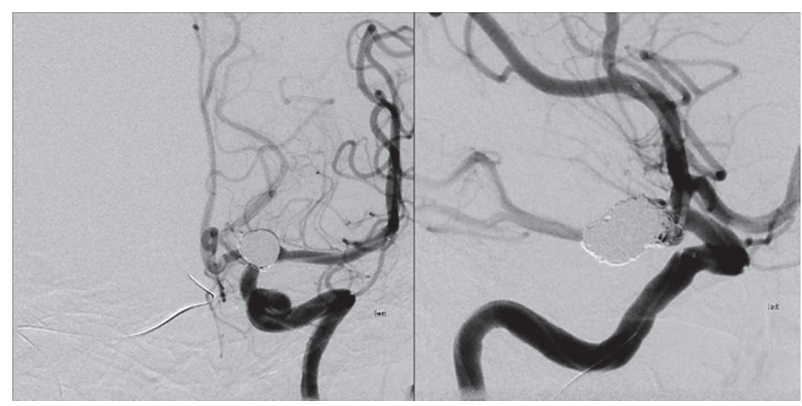

Abstract E-036 Figure 1

tool used in these cases, providing access to the target vessel through the contralateral or opposite circulation when anterograde access is difficult or non-achievable.

Materials and methods Cases of transcirculation intracranial interventions performed between two institutions (Tampa General Hospital, Tampa, FL and University of Texas Medical Branch, Galveston, TX) were reviewed, which included four cases of aneurysm and arteriovenous malformation (AVM) embolization. Complications, angiographic results, and clinical outcomes were assessed.

Results Successful transcirculation neurointerventions were performed in four cases. These cases include a posterior communicating (Pcomm) artery aneurysm which was coiled with M1 to Pcomm stent assistance, a basilar tip aneurysm which was coiled with P1 to contralateral P1 stent assistance, a complex posterior fossa AVM, and a giant left cavernous internal carotid artery (ICA) aneurysm. The posterior fossa AVM was embolized through a recurrent superior cerebellar artery retrogradely catheterized via the contralateral posterior communicating artery. The left cavernous ICA aneurysm was initially unsuccessfully treated with an anterograde approach and was retreated with a combined anterograde and retrograde approach through the contralateral anterior circulation across the anterior communicating artery with subsequent successful positioning of multiple flow diverters. In three cases the anterior or posterior circulation were accessed through the posterior communicating arteries, and in one case the contralateral anterior circulation was accessed by crossing the anterior communicating artery.

Conclusions Transcirculation approaches can be performed for cases where conventional treatment options are not feasible or as a bailout strategy in failed treatment attempts. In this small case series, it seemed a safe and efficient way of facing challenging cases.

Disclosures M. Colasurdo: None. G. Edhayan: None. P. Kan: None. K. Raghuram: None.

\section{E-037 ANGIOGRAPHIC INVESTIGATION OF ORBITAL VASCULAR VARIATIONS IN THE RABBIT: IMPLICATIONS FOR INTRA- ARTERIAL CHEMOTHERAPY MODELS}

${ }^{1} \mathrm{M}$ Feldman*, ${ }^{2} \mathrm{~B}$ Poitras, ${ }^{3} \mathrm{~A}$ Daniels, ${ }^{1} \mathrm{M}$ Froehler. ${ }^{1}$ Neurosurgery, Vanderbilt University Medical Center, Nashville, $T_{N}{ }^{2}$ Radiology, Vanderbilt University, Nashville, $T N_{;}{ }^{3}$ Department of Ophthalmology and Visual Sciences, Vanderbilt University Medical Center, Nashville, TN

\subsection{6/neurintsurg-2021-SNIS.133}

Introduction/Purpose The New Zealand White rabbit (NZWR) is the first small-animal experimental model of intra-arterial chemotherapy (IAC) for retinoblastoma treatment. The NZWR has dual ophthalmic arteries (OA): the external OA (EOA) arises from the external carotid artery and the internal OA (IOA) from the internal carotid artery. We describe the technique that we have refined for $\mathrm{OA}$ catheterization in rabbits, and describe the angioanatomical variations in the $\mathrm{OA}$ supply to the NZWR eye and implications for IAC delivery, which were identified as part of a project exploring IAC effects in a rabbit retinoblastoma model.

Materials and Methods We developed a novel technique to perform angiography of the external and internal carotid arteries and superselective angiography of the EOA and IOA in NZWR using transfemoral access and a microwire/microcatheter system that closely resembled our human IAC paradigm. EOA and IOA supply to the eye was determined angiographically and recorded before selective OA catheterization and angiography.

Results 114 rabbits underwent carotid angiographic evaluation and OA catheterization (161 total eyes evaluated, 112 right, 49 left). Most eyes had a single dominant arterial supply; either IOA or EOA. EOA was dominant in 73\% (118/161), and IOA was dominant in $17 \%(27 / 161)$. Co-dominant supply was seen in $10 \%(16 / 161)$. Of the rabbits with bilateral OA catheterization, 25/47 (53\%) had bilateral dominant EOA.

Conclusions Successful catheterization of the $\mathrm{OA}$ in the NZWR can be readily accomplished with nuanced technique that mimics that used in humans. The external $O A$ is the dominant arterial supply in the majority of NZWR eyes. These findings allow for successful reproduction of OA catheterization studies of IAC for retinoblastoma in NZWR. These findings may also give insight into ICA and ECA-targeting IAC techniques in humans.

Disclosures M. Feldman: None. B. Poitras: 5; C; Cerenovus. A. Daniels: None. M. Froehler: 1; C; Genentech, Medtronic, Stryker, Microvention, and Penumbra. 2; C; Genentech, Medtronic, Stryker, Balt USA, Viz.ai, and Corindus.

\section{E-038 THROMBECTOMY OUTCOMES AND OBSERVATIONS ON DENTAL HYGIENE (TOOTH)}

${ }^{1} \mathrm{M}$ Feldman*, ${ }^{2} \mathrm{~S}$ Koester, ${ }^{3} \mathrm{R}$ Chaliff, ${ }^{1} \mathrm{M}$ Longo, ${ }^{1} \mathrm{~A}$ Yengo-Kahn, ${ }^{4} \mathrm{M}$ Froehler, ${ }^{1} \mathrm{R}$ Chitale. ${ }^{1}$ Neurosurgery, Vanderbilt University Medical Center, Nashville, $T N_{i}{ }^{2}$ Vanderbilt University School of Medicine, Nashville, TN; ${ }^{3}$ Oral and Maxillofacial Surgery, Vanderbilt University Medical Center, Nashville, TN; ${ }^{4}$ Cerebrovascular Diseases Program, Vanderbilt University Medical Center, Nashville, TN

\subsection{6/neurintsurg-2021-SNIS.134}

Introduction Previous literature has identified an association between poor dentition and risk for stroke. This association has not been identified for acute stroke outcomes after endovascular treatment. We aimed to assess whether tooth loss and dental status was predictive of poor outcomes after thrombectomy for acute ischemic stroke.

Methods A retrospective review was conducted on all adult patients at a single comprehensive stroke center who underwent thrombectomy between 2012 to 2018. Inclusion criteria included complete CT imaging where dentition could be assessed for the front 28 teeth. Demographic, comorbidity, and outcome data were also collected for all of these patients. A multivariate analysis was performed, with the primary outcome as post-operative MRS.

Results 273 consecutive patients were included in our analysis. The average age was $62.96(+/-15.76)$ and $137(50 \%)$ of the patients were male. Patients missing all teeth did not 